Supporting Information

\title{
Mesoporous Polymer-Derived Ceramic Membranes for Water Purification via a Self-Sacrificed Template
}

Zewen Zhang, ${ }^{\dagger}, \S$ Yueping Bao, ${ }^{£ \S}$ Xun Sun, ${ }^{\&}$ Ke Chen, ${ }^{\star}$ Mingjiong Zhou, ${ }^{*}$ Liu He, ${ }^{\ddagger}$ Qing Huang, ${ }^{*}$ Zhengren Huang, ${ }^{*}$ Zhifang Chai, $*$ and Yujie Song $* *$ 


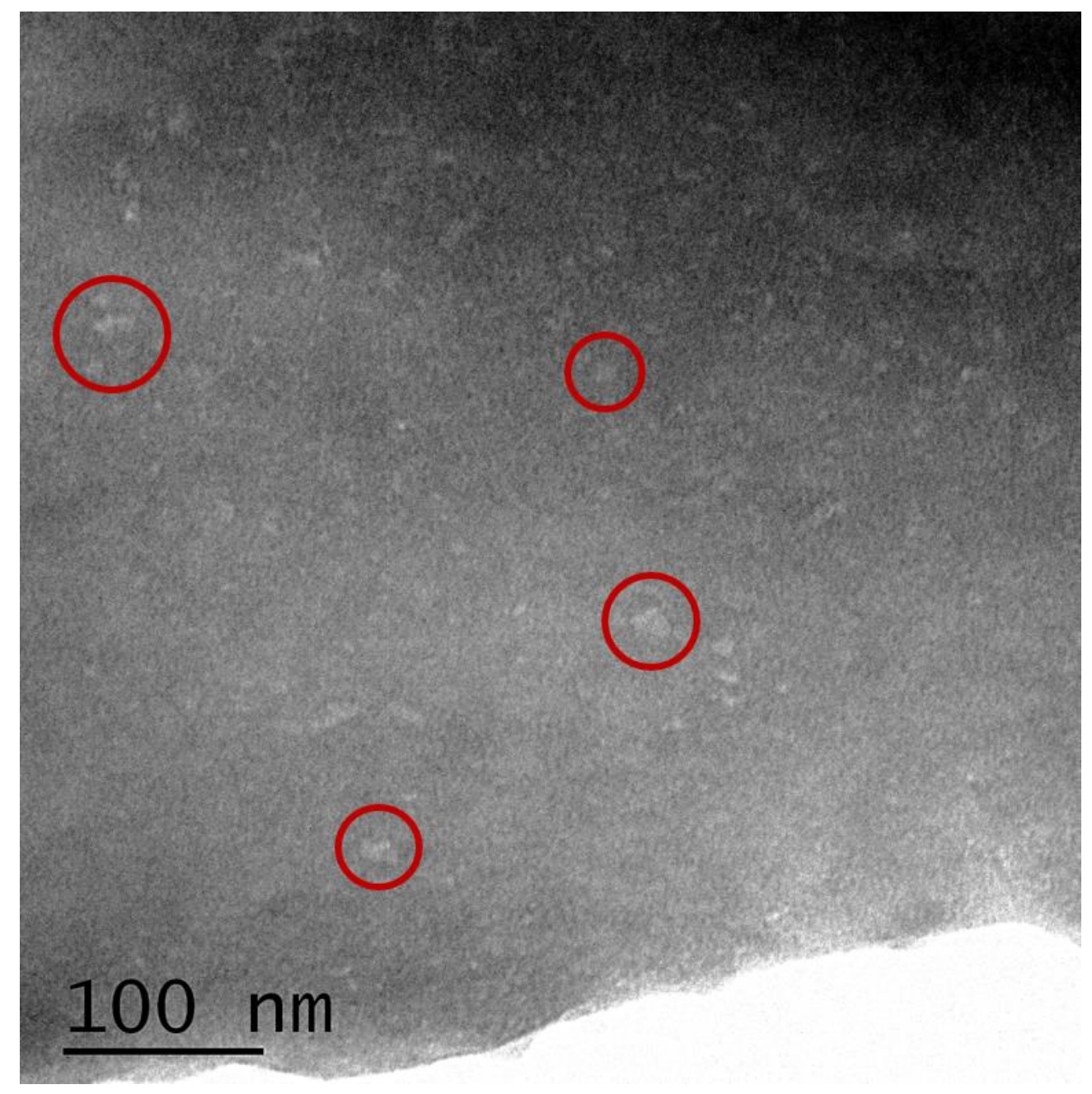

Figure S1. TEM image of nanosized PDMS region in cyclosiloxane hybrid polymer. The white regions marked with red circles are phase separated PDMS (30 wt $\left.\%, \mathrm{Mw}=63,000 \mathrm{~g} \mathrm{~mol}^{-1}\right)$. 


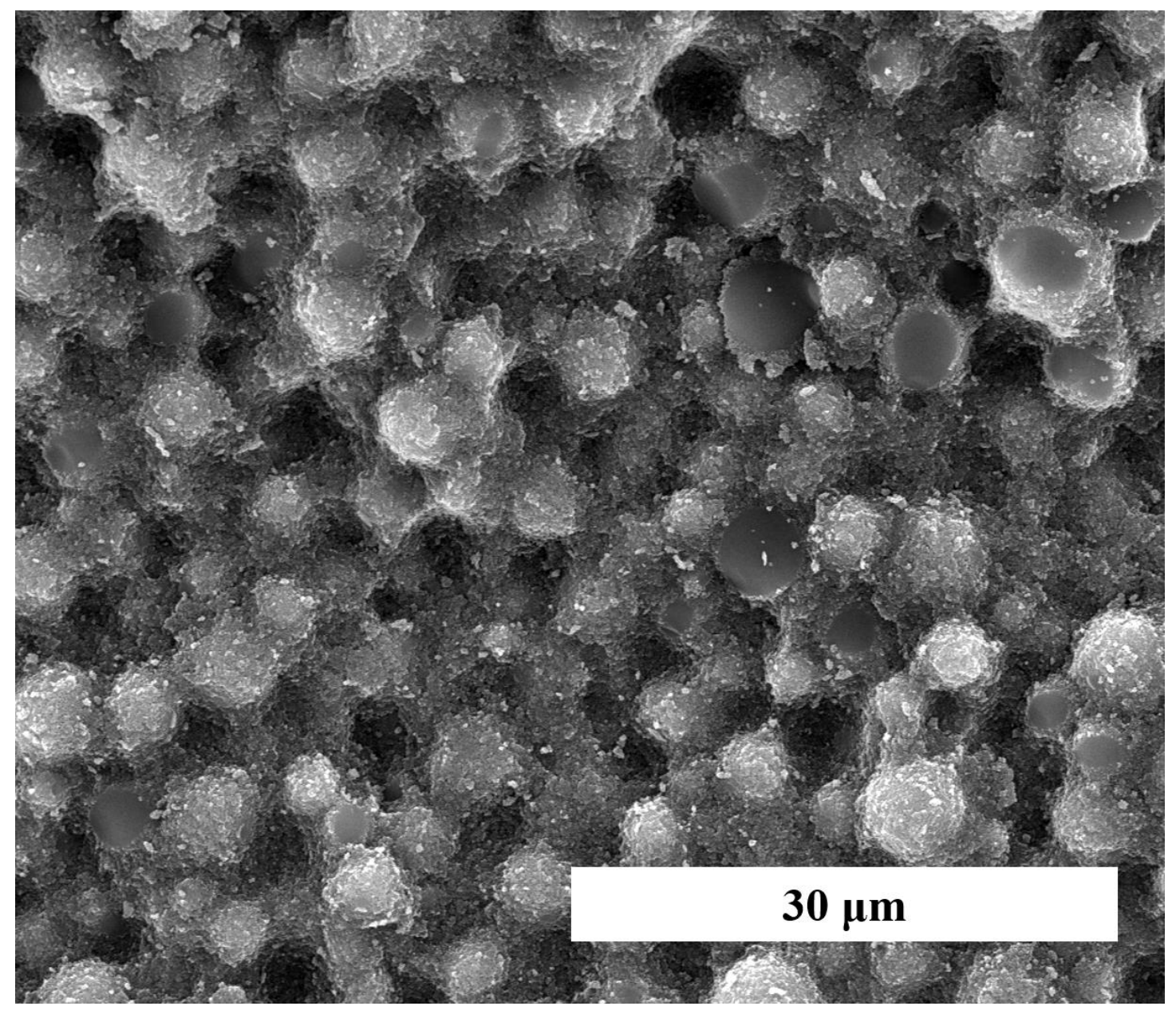

Figure S2. SEI image of SiOC membrane prepared with $20 \mathrm{wt} \%$ PDMS (vinyl terminated, Mw

$$
\left.=25,000 \mathrm{~g} \mathrm{~mol}^{-1}\right) \text {. }
$$




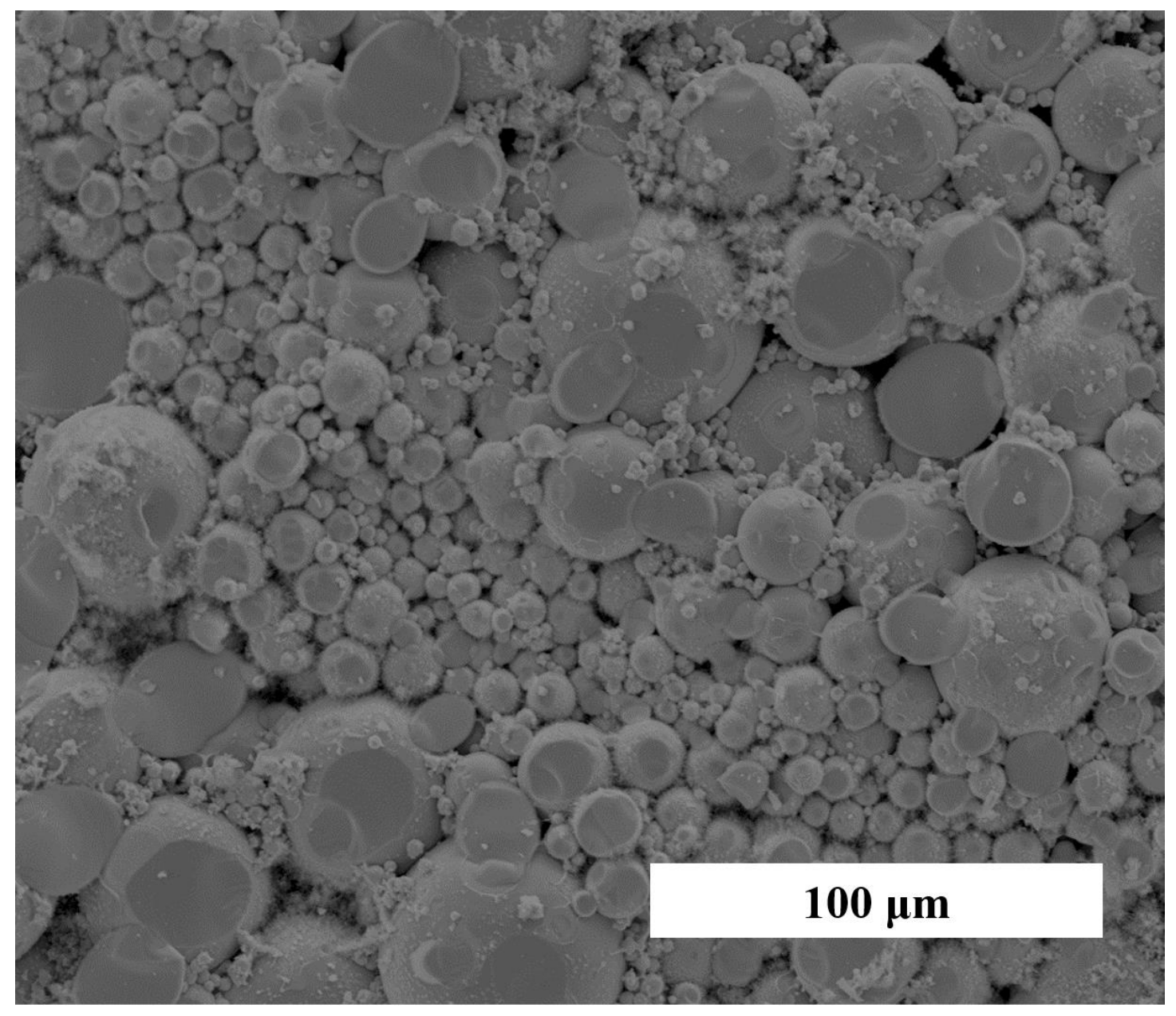

Figure S3. SEI image of SiOC membrane prepared with $10 \mathrm{wt} \%$ PDMS (trimethryl terminated, $\left.\mathrm{Mw}=25,000 \mathrm{~g} \mathrm{~mol}^{-1}\right)$. 


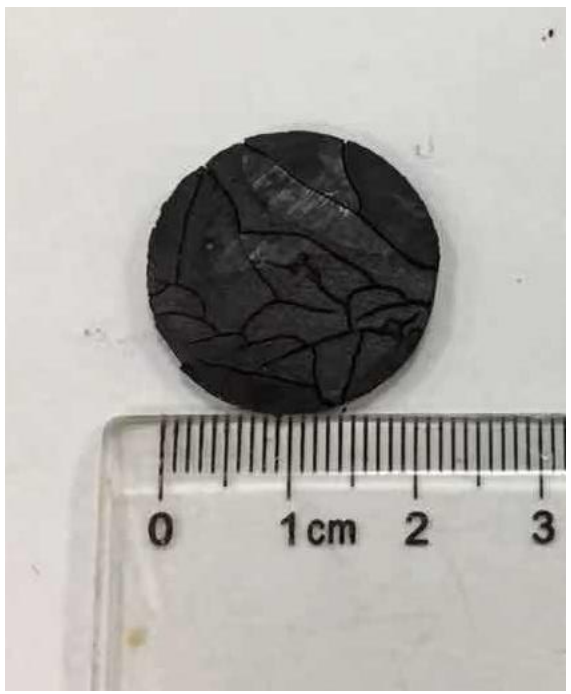

Figure S4. Digital image of sintered SiOC membrane prepared with $30 \mathrm{wt} \%$ vinyl terminated PDMS.

(Photograph courtesy of 'Yujie Song'. Copyright 2020. Free domain.) 

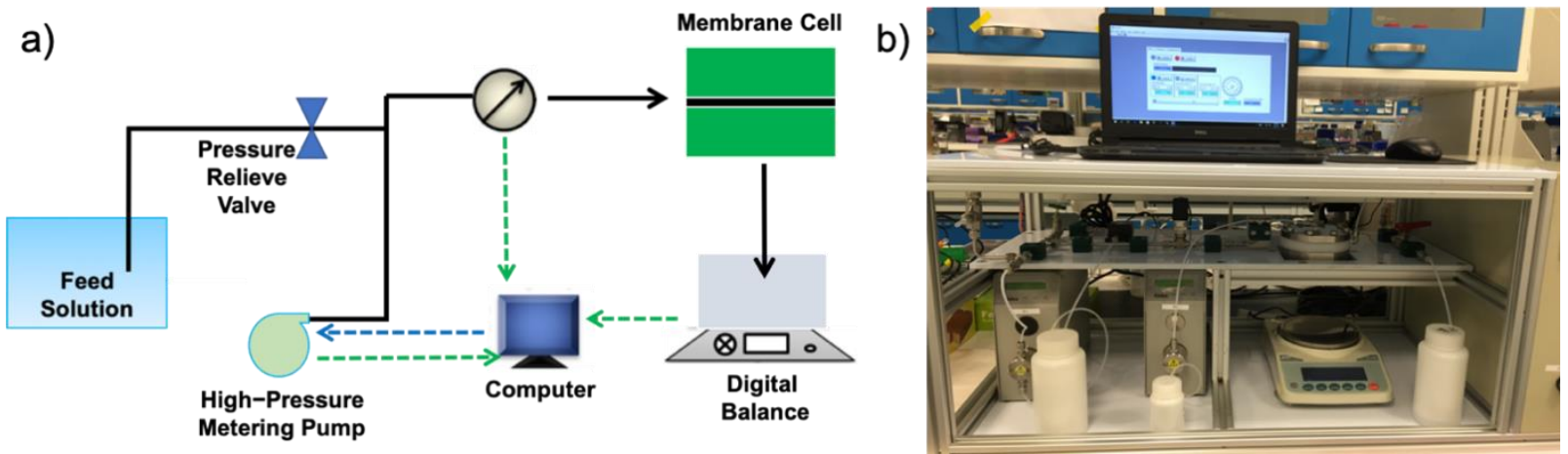

Figure S5. The schematic diagram (a) and digital image (b) of the home-made dead-end membrane filtration system.

(Photograph courtesy of 'Yueping Bao'. Copyright 2020. Free domain.) 